\title{
Thyroid screening of members of the public for iodine isotopes with portable $\mathrm{NaI}$ detectors
}

\author{
J. G. Hunt \\ Instituto de Radioproteção e Dosimetria -(CNEN) Av. Salvador Allende s/n 22780-160 Rio de Janeiro - RJ, Brazil \\ john@ird.gov.br
}

\begin{abstract}
In the case of an accident in a nuclear power plant with radionuclide releases to the environment, members of the public with possible internal contamination with radioactive isotopes of iodine should be screened to identify cases where a more detailed evaluation and medical follow-up is necessary. Screening of large numbers of the public can be performed with a quick measuring protocol using hand held unshielded $\mathrm{NaI}$ based detectors giving results in cps. The screening geometry was simulated using the Monte Carlo code Visual Monte Carlo. The results show that for a geometry with the NaI detector near the skin in front of the thyroid, the interference of the gamma radiation coming from other radionuclides released in the accident either deposited in the lung or in the whole body is sufficiently low to allow thyroid screening criteria to be established. The screening criteria were developed using 5, 10 and 15 year old hybrid phantoms and for the adult male based on the ICRP reference voxel phantom.
\end{abstract}

Keywords:

Emergency screening, Monte Carlo, voxel phantoms

\section{INTRODUCTION}

The massive release of radionuclides from a nuclear power plant (NPP) can result in the intake of fission products by members of the public and emergency workers. The resulting body burdens may reach levels where medical treatment and follow-up is required. As part of the emergency response planning, members of the public who may have inhaled or ingested the radionuclides should be screened for internally deposited radionuclides (IAEA, 2004). 
The protocol for the screening of the public should take into consideration that the measurements must be made quickly and large numbers of people need to be screened. Portable hand-held equipment is readily available. The objective of the screening is to determine which individuals should be indicated for further internal individual monitoring and possible medical treatment. The Goiania accident with ${ }^{137}$ Cs (IAEA, 1988) and the Fukushima accident (UNSCEAR 2013) are examples of this necessity.

In this paper, the internal contamination of individuals with a wide range of radionuclides such as that seen after a large scale accident at a nuclear Power plant (NPP) is simulated, and the measurement in counts-per-second (cps) by unshielded hand-held equipment is simulated using the Monte Carlo technique.

\section{MATERIALS AND METHODS}

Four phantoms were used for this study that represent the various age groups of the public. The voxel phantoms used were the ICRP male reference phantom (ICRP, 2009) and the 5, 10 and 15 year old male hybrid phantoms produced by the University of Florida (Lee, 2010). Figure 1 shows the "family" of hybrid phantoms.

Figure 1. The University of Florida family of 5, 10 and 15 year old phantoms

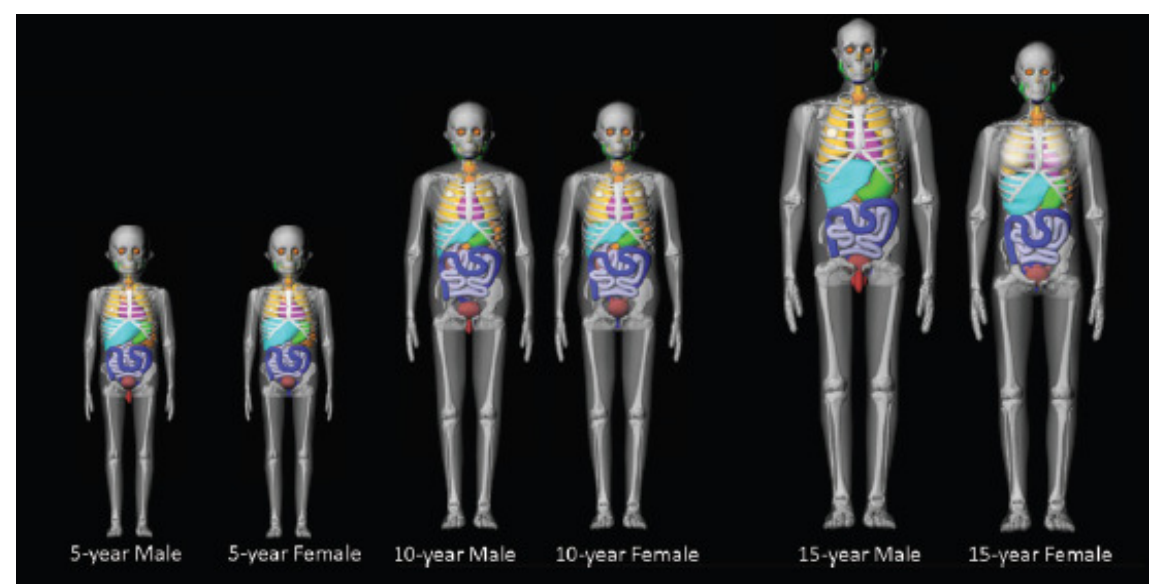


The Monte Carlo program used is Visual Monte Carlo (VMC) in-vivo, available for free download from http://www.vmcsoftware.com/Index.html (Hunt, 2002) and Gomes-Rós, 2010). The emission of photons by fission products in the thyroid, lung and the whole body were simulated. The photons were transported through the phantom and then detected in a $\mathrm{NaI}$ detector. Instead of binning counts in the photopeak, as is usually the case in gamma spectrometry, the total cps in the NaI crystal was simulated. If the photon suffered one or more Compton interactions or a photoelectric interaction in the NaI, then one count was added to the total counts.

The detector used in this paper was the Identifinder NHG Ultra with a NaI crystal with $35 \mathrm{~mm}$ diameter and $51 \mathrm{~mm}$ height. The same work has also been performed in a more complete study by this author using $\mathrm{NaI}$ detectors of geometry 1" x 1" (2.54 mm diameter x $2.54 \mathrm{~mm}$ height) and 2" x 2" (5.08 mm diameter x $5.08 \mathrm{~mm}$ height) as seen in hand-held equipment such as the Canberra SG-1R or SG-2R. The NaI crystals were not considered to be shielded or collimated. During a severe accident at a NPP, a number of radionuclides are emitted, some of which have the thyroid as the critical organ, some of which are deposited and remain in the lung while others are quickly transported to the soft tissues

\section{RESULTS}

\subsection{Calibration Factors for Iodine Isotopes in the Thyroid}

Simulations were run for the four phantoms with ${ }^{131} \mathrm{I},{ }^{132} \mathrm{I},{ }^{133} \mathrm{I}$ and ${ }^{135} \mathrm{I}$ in the thyroid. The results for the Identifinder are given in Table 1 and the graphics are shown in Figure 2. 
Table 1. Calibration factors for radio-iodines in the thyroid measured with an Identifinder placed over the thyroid and at a distance of $0.2 \mathrm{~cm}$ from the skin.

Calibration factors (cps/kBq)

\begin{tabular}{ccccc}
\hline Radionuclide & $\mathbf{5} \mathbf{y}$ & $\mathbf{1 0} \mathbf{y}$ & $\mathbf{1 5} \mathbf{y}$ & Adult \\
\hline I-131 & 57 & 56 & 48 & 33 \\
I-132 & 120 & 119 & 103 & 71 \\
I-133 & 105 & 102 & 85 & 53 \\
I-135 & 43 & 43 & 37 & 26 \\
\hline
\end{tabular}

The 5 and 10 year phantoms show similar values of cps. The ICRP adult phantom shows lower calibration factors as the thyroid in this phantom is deeper below the skin surface.

Figure 2. Simulation of the Identifinder placed over the thyroid of the 5 year old male detecting photons emitted from ${ }^{131}$ I

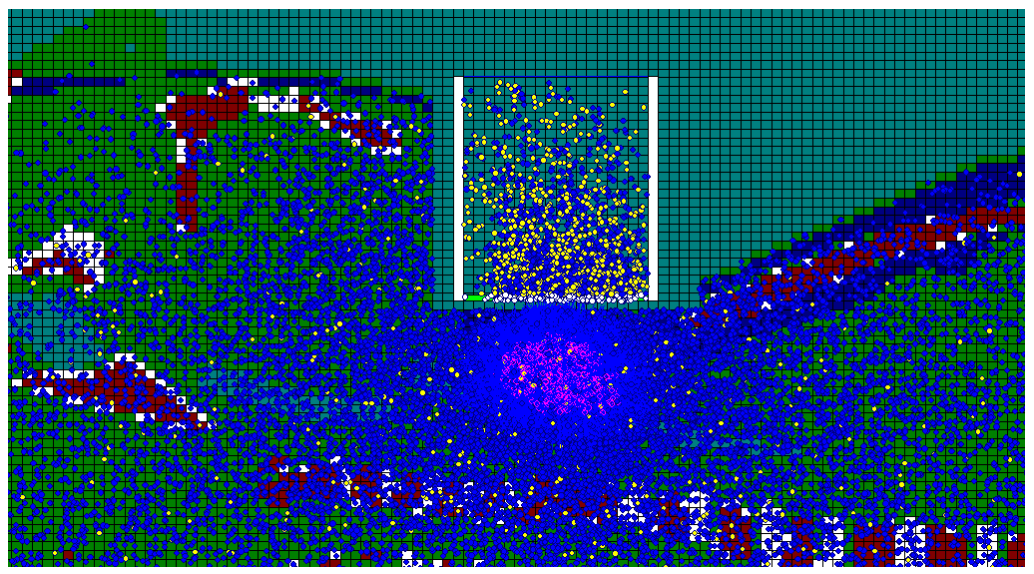

\subsection{Calibration Factors for Whole Body Radionuclides Counted at the Thyroid}

The presence of ${ }^{134} \mathrm{Cs},{ }^{136} \mathrm{Cs}$ and ${ }^{137} \mathrm{Cs}$ in the whole body causes a "background" or "cross-fire" cps in the NaI detector positioned at the thyroid. Simulations were carried out for the ICRP male adult to determine some values for the "cross-fire". The results are given in Table 2. 
Table 2. Calibration factors for a cesium contamination of muscle, fat and soft tissues (whole body) with the Identifinder placed over the thyroid for the adult phantom

\begin{tabular}{cc}
\cline { 2 - 2 } & $\begin{array}{c}\text { Calibration factor } \\
(\mathrm{cps} / \mathrm{kBq})\end{array}$ \\
\hline Radionuclide & Adult \\
\hline Cs-134 & 1.8 \\
Cs-136 & 2.4 \\
Cs-137 & 0.69 \\
\hline
\end{tabular}

\subsection{Calculation of Interference of Lung Radionuclides on Measurements at Thyroid}

The radionuclides deposited in the lung are relatively close to the detector at the thyroid position and will cause a measurable "cross-fire". In order to determine the magnitude of this cross-fire, the contamination of the lung was simulated and the cps evaluated in the detector placed at the thyroid, see Table 3 and Figure 3. 
Table 3. Calibration factors for radionuclides in the lung with an Identifinder placed over the thyroid

\begin{tabular}{ccccc} 
& \multicolumn{4}{c}{ Calibration factors (cps/kBq) } \\
\cline { 2 - 5 } Radionuclide & $\mathbf{5}$ y & $\mathbf{1 0} \mathbf{y}$ & $\mathbf{1 5} \mathbf{y}$ & Adult \\
\hline Zr-95 & 4.9 & 3.9 & 3.7 & 3.1 \\
Mo-99 & 7.7 & 5.5 & 5.2 & 4.3 \\
Ru-103 & 5.7 & 4.3 & 4.2 & 3.4 \\
Ru-105 & 6.7 & 5.3 & 4.8 & 3.9 \\
Te-127m & 0.30 & 0.13 & 0.12 & 0.13 \\
Te-129m & 0.24 & 0.09 & 0.07 & 0.10 \\
Te-131m & 21 & 16 & 14 & 12 \\
Te-132 & 13 & 9.4 & 8.2 & 6.9 \\
Ba-140 & 13 & 9.4 & 9.4 & 7.5 \\
Ce-141 & 4.6 & 3.2 & 3.0 & 2.5 \\
Ce-143 & 6.5 & 4.6 & 4.2 & 3.5 \\
\hline
\end{tabular}


Figure 3. Simulation of gamma radiation emitted from ${ }^{99} \mathrm{Mo}$ in the lungs, with the detector placed at the thyroid position of the 5, 10 and 15 year male.

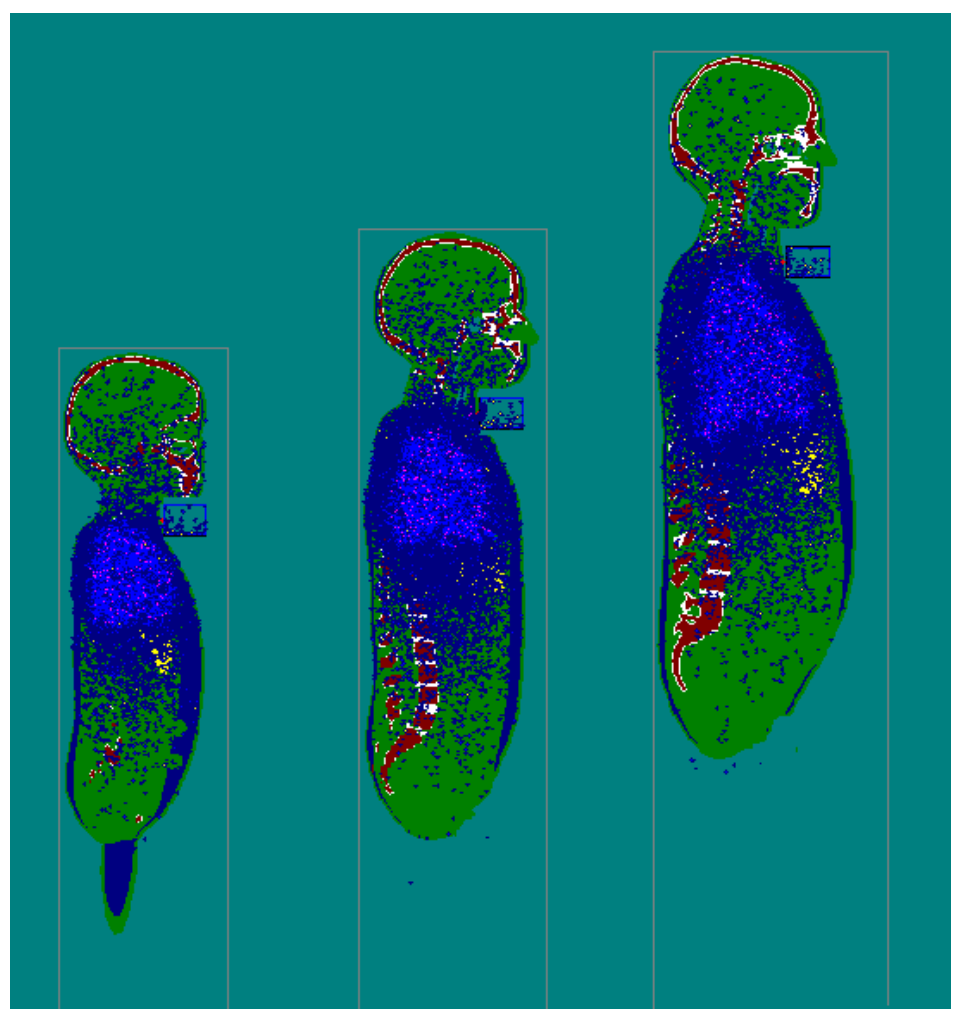

\subsection{Release of radionuclides in the case of a severe nuclear power plant accident}

In the case of a severe NPP accident, a mixture of isotopes of iodine, cesium and other fission products are released. Taking as an example the radionuclides released during the Chernobyl accident and the release fractions established in UNSCEAR as reference (UNSCEAR, 2006), and assuming for a given member of the public a ratio of inhaled activity to released activity of $10^{-15}$ the end result is as given in Table 5, assuming that all the radio-iodines inhaled end up in the thyroid. 
Table 5. Total cps emitted due to the radio-iodines in the thyroid, the radio-cesium being deposited in the whole body, and the insoluble radionuclides being deposited in the lungs, for the 5, 10, 15 and adult phantoms, with the detector placed at the thyroid.

\begin{tabular}{|c|c|c|c|c|c|c|c|}
\hline \multirow{2}{*}{ Radionuclide } & \multirow{2}{*}{$\begin{array}{c}\text { Released } \\
\text { PBq }\end{array}$} & \multirow{2}{*}{$\begin{array}{c}\text { Inhaled } \\
\text { kBq }\end{array}$} & \multirow{2}{*}{$\begin{array}{c}\text { Tissue or } \\
\text { organ }\end{array}$} & \multicolumn{4}{|c|}{ cps at thyroid with Identifinder } \\
\hline & & & & $5 y$ & $10 y$ & $15 y$ & adult \\
\hline $\mid-131$ & 1760 & 1.76 & Thyroid & 100 & 99 & 84 & 58 \\
\hline \multirow[t]{2}{*}{ I-133 } & 910 & 0.91 & Thyroid & 96 & 93 & 77 & 48 \\
\hline & & & subtotal & 196 & 192 & 161 & 106 \\
\hline Cs-134 & 47 & 0.047 & Soft tissues & & & & 0.08 \\
\hline Cs-136 & 36 & 0.036 & Soft tissues & & & & 0.09 \\
\hline \multirow[t]{2}{*}{ Cs-137 } & 85 & 0.085 & Soft tissues & & & & 0.06 \\
\hline & & & subtotal & & & & 0.23 \\
\hline Zr-95 & 84 & 0.084 & Lung & 0.41 & 0.33 & 0.31 & 0.26 \\
\hline Mo-99 & 100 & 0.1 & Lung & 0.77 & 0.55 & 0.52 & 0.43 \\
\hline Ru-103 & 200 & 0.2 & Lung & 1.139 & 0.87 & 0.83 & 0.68 \\
\hline Te-129m & 240 & 0.24 & Lung & 0.057 & 0.022 & 0.017 & 0.024 \\
\hline Te-132 & 1150 & 1.15 & Lung & 15 & 11 & 9 & 8 \\
\hline Ba-140 & 240 & 0.24 & Lung & 3.1 & 2.2 & 2.2 & 1.8 \\
\hline \multirow[t]{2}{*}{ Ce-141 } & 84 & 0.084 & Lung & 0.39 & 0.27 & 0.25 & 0.21 \\
\hline & & & subtotal & 20 & 15 & 14 & 11 \\
\hline
\end{tabular}

The following conclusions may be derived from Table 5: (a) for the same activity of radioiodine in the thyroid, the cps measured for the 5 and 10 year olds is double that of the adult, due to the fact that the thyroid is closer to the skin surface for these two ages. (b) The radioisotopes of cesium distributed over the whole body do not contribute to the cps measured by the Identifinder located at the thyroid. Not only is the inhaled activity much lower for the radio-cesium, but also the spread of the radio-cesium over the whole body significantly reduces the calibration factors in $\mathrm{cps} / \mathrm{kBq}$. (c) The radionuclides deposited in the lung contribute around $10 \%$ of the cps measured by the Identifinder located at the thyroid in relation to the direct count of the iodines deposited in the thyroid, for all ages. (d) The inhalation of $1.76 \mathrm{kBq}$ of ${ }^{131} \mathrm{I}$ will lead to an 
equivalent dose to the thyroid of approximately $0.4 \mathrm{mSv}$ and the inhalation of $0.91 \mathrm{kBq}$ of ${ }^{133} \mathrm{I}$ will lead to an equivalent dose to the thyroid of approximately $0.05 \mathrm{mSv}$ for type $\mathrm{F}$ and $5 \mu \mathrm{m}$ AMAD. For the adult, the inhaled activity required to produce a $100 \mathrm{mSv}$ equivalent dose to the thyroid would show around 30,000 cps on the Identifinder at the thyroid. The Identifinder maximum range is $100,000 \mathrm{cps}$.

\section{VALIDATION OF THE METHOD}

The Monte Carlo calculations used in this paper were validated using the thyroid phantom developed at the IRD which had been used previously as part of an IAEA regional intercomparison exercise for in-vivo thyroid measurement (Dantas, 2011). ${ }^{133} \mathrm{Ba}$ was used as a substitute for ${ }^{131} \mathrm{I}$ as it has a photo-peak at almost exactly the same energy as ${ }^{131} \mathrm{I}(356 \mathrm{keV})$ and a half-life of 10.5 years.

The ICRP male phantom was used in VMC, and the head and thorax was removed so as to simulate as far as possible the cylindrical geometry of the thyroid phantom as shown in Figure 4. The ${ }^{133} \mathrm{Ba}$ in the thyroid was measured with the Identifinder NHG-ULTRA at three distances and the results are given in Table 6.

Figure 4. Simulation of Identifinder placed over thyroid of adult phantom counting ${ }^{133} \mathrm{Ba}$ in the thyroid.

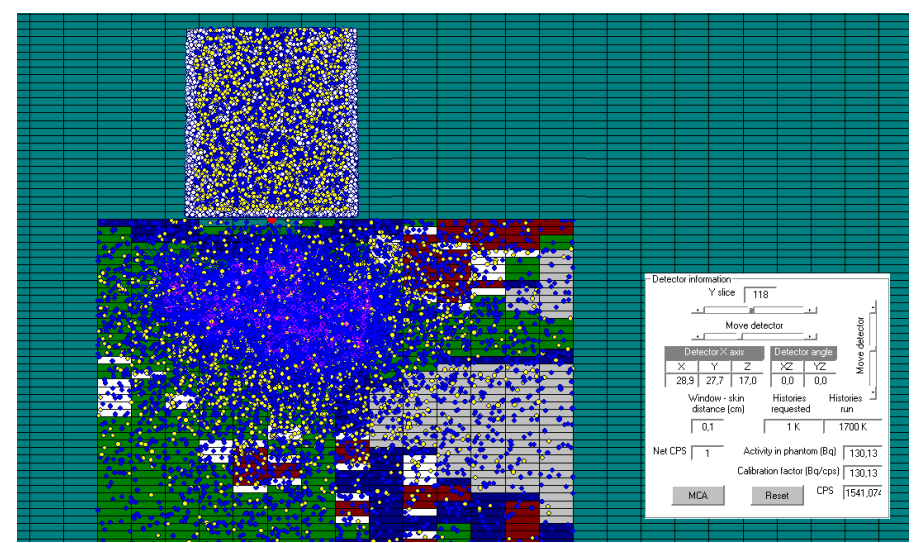


Table 6. Validation results for ${ }^{133} \mathrm{Ba}$ in the thyroid with an Identifinder placed at three distances from the skin surface.

\begin{tabular}{cccc}
\hline $\begin{array}{c}\text { Distance to surface } \\
\text { thyroid phantom } \\
\text { (cm) }\end{array}$ & $\begin{array}{c}\text { Identifinder } \\
\text { measurement } \\
\text { (net cps) }\end{array}$ & $\begin{array}{c}\text { VMC } \\
\text { calculation } \\
\text { (cps) }\end{array}$ & $\begin{array}{c}\text { Ratio of Identifinder cps to } \\
\text { VMC cps }\end{array}$ \\
\hline 0.1 & 1200 & 1310 & 0.92 \\
4.8 & 410 & 366 & 1.1 \\
9.8 & 170 & 153 & 1.1 \\
\hline
\end{tabular}

A loss of signal through the photomultiplier and electronics of the Identifinder of $85 \%$ was considered in this paper. An electronic efficiency for NaI detectors between around $75 \%-90 \%$ has been verified in previous VMC validation work.

The ICRP male phantom thyroid and the thyroid phantom are different in their geometry as the phantom thyroid is represented by a sheet of paper cut into a shape resembling a butterfly, which is placed into a tissue equivalent plastic cylinder resembling the human neck.

\section{UNCERTAINTIES}

There are three main sources of uncertainty:

a) The highest source of uncertainty is the difference between the phantom used and the real person. The uncertainty in the calculation of the calibration factors is estimated to be around $\pm 20 \%$ for the thyroid and $\pm 30 \%$ for the lung and whole body counting.

b) The second source of uncertainty is the NaI detector "electronic efficiency". For the Identifinder, this is estimated to be $85 \%$. That is, for every 100 photons that cause a Compton or photoelectric interaction in the $\mathrm{NaI}$ crystal, 85 photons are detected by the photo-multiplier and converted into one "count" on the detector. The variation of this "electronic efficiency" between detectors of the same model and manufacturer is estimated to be around $\pm 15 \%$.

c) The Monte Carlo method employed is subject to a maximum uncertainty of $\pm 10 \%$

Compiling these uncertainties leads to a propagated uncertainty of around $40 \%$. 


\section{CONCLUSIONS}

It is possible to estimate the activity of the radio-iodines in the thyroid with an unshielded NaI detector. The cross-fire cps coming from radionuclides deposited in the lung and whole body is around $10 \%$ of the cps coming from the iodine isotopes in the thyroid for all ages.

Calibration factors have been calculated for the Identifinder NHG Ultra with a NaI crystal with $35 \mathrm{~mm}$ diameter and $51 \mathrm{~mm}$ height, and other NaI crystal geometries have also been simulated. NaI crystals of this size already show very high cps $(>10,000)$ for thyroid activities which would cause an equivalent dose above $100 \mathrm{mSv}$. Possibly a $25.4 \mathrm{~mm}$ x $25.4 \mathrm{~mm}$ NaI detector would be the most indicated, or a thin $\mathrm{NaI}$ detector such as a $9 \mathrm{~mm}$ diameter $\mathrm{x} 2 \mathrm{~mm}$ thick crystal.

\section{ACKNOWLEDGEMENTS}

The author would like to thank Mr. Choonsik Lee and the University of Florida for the use of the University of Florida hybrid voxel phantoms.

\section{REFERENCES}

Dantas B.M., Dantas A.L.A, Santos D.S., Cruz-Suarez R. IAEA Regional Intercomparison of in vivo Measurements Of 131-I in the Thyroid: The Latin American and Caribbean experience. Radiat. Prot. Dosimetry 144, pp.291-294, 2011.

Gomes-Ros, J., Hunt J. et al. Monte Carlo modeling of Germanium detectors for the measurement of low energy photons in internal dosimetry: Results of an international comparison. Radiation Measurements, 43, pp.510 - 515, 2008.

IAEA, Development of an extended framework for emergency response criteria, IAEATECDOC-1432, Vienna, 2004.

IAEA, The radiological accident in Goiania, Vienna, 1988. 
ICRP. Adult Reference Computational Phantoms. ICRP Publication 110. Ann. ICRP 3922009.

Lee C., Lodwick, D., Hurtado, D. Pafundi, D., Williams J., Bolch W. The UF family of reference hybrid phantoms for computational radiation dosimetry, Phys. Med. Biol. 55 pp.339-363, 2010.

UNSCEAR 2006 report Volume 1, Annex A, Epidemiological studies of Radiation and Cancer.

UNSCEAR 2013 report Volume I: Annex A: Levels and effects of radiation exposure to the nuclear accident after the 2011 great east-Japan earthquake and tsunami. 